\title{
ANALYTIC CONTINUATION OF THE S-MATRIX FOR POTENTIAL SCATTERING
}

\author{
JAMES S. HOWLAND
}

In this note, we propose to give shortened proofs of two theorems of Dolph, McLeod and Thoe [4] on the analytic continuation of the resolvent kernel and $S$-matrix for potential scattering to the "unphysical sheet." The proofs will use Carleman's inequality and the currently fashionable method of factoring the potential. (See [5], [9] and the references given there.) Since our purpose is to illustrate a method, we shall consider the problem in dimension three only, although McLeod [10] has extended the results of [4] to arbitrary dimensions.

The author wishes to thank Professor James Rovnyak for several conversations.

$R_{\mathbf{3}}$ will denote real Euclidean three-space and $\Delta$ the Laplacian operator on $L_{2}\left(R_{3}\right)$. Integrals without an indicated domain of integration are over all of $R_{3}$.

We shall adopt the hypotheses of [4].

Assumption. Let $V(x)$ be a real-valued function on $R_{3}$ such that

(1) $V(x)$ is locally Holder continuous except for a finite number of square integrable singularities, and

(2) there exist positive constants $C, \alpha$ and $R$ such that

$$
|V(x)| \leqq C e^{-\alpha|x|} \quad \text { for }|x| \geqq R .
$$

The Schroedinger operator $T=-\Delta+V(x)$ is then selfadjoint with domain $D(T)=D(\Delta)$ [8].

LEMma 1. If $f, g \in L_{2}\left(R_{3}\right)$, then $\iint|f(x) g(y)||x-y|^{-2} d y d x<+\infty$.

This is an immediate consequence of Sobolev's inequality $[1, p$. 220, part (c)].

The following fact is well known, but, having no convenient reference for it, we supply a brief proof.

Lemma 2. Let $(\mu, X)$ be a measure space and $f(\cdot, k) \in L_{2}(\mu, X)$ for each $k$ in a domain $\Omega$ of the complex plane. Assume that for a.e. $x$ in $X$, $f(x, k)$ is analytic on $\Omega$ with $k$-derivative $f^{\prime}(x, k)$. If $|f(x, k)| \leqq g(x)$ for some $g \in L_{2}(\mu, X)$, then $f(\cdot, k)$ is a vector-valued analytic function on $\Omega$ and its derivative is $f^{\prime}(\cdot, k)$.

Received by the editors May 27, 1968.

${ }^{1}$ Partly supported by ARO Grant DA-ARO-D-31-124-G978. 
Proof. If $k$ is fixed and $\Omega$ contains $\left\{z:|z-k| \leqq 2 r_{0}\right\}$, then for $|h| \leqq r_{0}$

$f(x, k+h)-f(x, k)=(h / 2 \pi i) \int_{|z-k|=2 r_{0}}(z-k)^{-1}(z-k-h)^{-1} f(x, z) d z$.

A simple estimate shows that $h^{-1}[f(\cdot, k+h)-f(\cdot, k)]$ is majorized by $\left(2 / r_{0}\right) g(x)$ for $|h| \leqq r_{0}$, and hence converges to $f^{\prime}(\cdot, k)$ in the norm of $L_{2}(\mu, X)$.

LemMa 3. Let $Q(k)$ be an operator-valued function analytic in HilbertSchmidt norm on a domain $\Omega$. If $I+Q(k)$ is invertible for some $k$ in $\Omega$, then $[I+Q(k)]^{-1}$ exists except at isolated points and is meromorphic on $\Omega$.

Proof. The points where $I+Q(k)$ is not invertible are the zeros of $\operatorname{det}_{2}[I+Q(k)]\left[3\right.$, p. 1106]. If $P_{n}$ is a sequence of finite rank orthogonal projections increasing strongly to $I$, then $P_{n} Q(k) P_{n} \rightarrow Q(k)$ in Schmidt norm for each $k$ in $\Omega$. The argument in the proof of part (c) of Lemma XI. 9.22 of [3] (pp. 1109-1110) shows that $\operatorname{det}_{2}[I+Q(k)]$ is analytic, so that $I+Q(k)$ is invertible except at isolated points. These points are poles of finite order by virtue of the Carleman inequality [3, Corollary 25, p. 1112$]$

$$
\left\|\left(\operatorname{det}_{2}[I+Q(k)]\right)[I+Q(k)]^{-1}\right\| \leqq \exp \left(C_{2}\|Q(k)\|_{2}^{2}\right)
$$

where $C_{2}$ is a constant.

Let $R_{0}(z)=(-\Delta-z)^{-1}$. If $\operatorname{Im} k>0$ and $k^{2}$ is in the resolvent set of $-\Delta$, then $R_{0}\left(k^{2}\right)$ is an integral operator with kernel

$$
G_{0}(x, y, k)=(1 / 4 \pi)|x-y|^{-1} e^{i k \mid x-y !} .
$$

Define $A(x)=e^{-\alpha|x| / 2}$ and $B(x)=e^{\alpha|x| / 2} V(x)$, and let $A$ and $B$ denote the corresponding multiplication operators. Note that $V(x)$ $=A(x) B(x)$.

TheOREM 1. (a) For Im $k>0, A R_{0}\left(k^{2}\right) B$ has a unique extension to a Hilbert-Schmidt operator $Q(k)$ on $L_{2}\left(R_{3}\right) . Q(k)$ is analytic for $\operatorname{Im} k>0$ and has an analytic continuation to $\operatorname{Im} k>-\alpha / 2$ in Hilbert-Schmidt norm.

(b) $I+Q(k)$ has a bounded inverse for $\operatorname{Im} k>-\alpha / 2$, except for isolated values of $k$. $[I+Q(k)]^{-1}$ is meromorphic on $\operatorname{Im} k>-\alpha / 2$, and the poles of $[I+Q(k)]^{-1}$ in $\operatorname{Im} k>0$ are pure imaginary, finite in number and occur when $k^{2}$ is a negative eigenvalue of $T$.

Proof. For $\operatorname{Im} k>0, A R_{0}\left(k^{2}\right) B$ has the kernel

$$
K(x, y, k)=(1 / 4 \pi)|x-y|^{-1} A(x) e^{i k|x-y|} B(y) .
$$


If $y \neq x$, (1) defines $K(x, y, k)$ as an entire function of $k$, which is majorized for $\operatorname{Im} k \geqq-\eta_{0}>-\alpha / 2$ by

$$
K_{0}(x, y)=(1 / 4 \pi)|x-y|^{-1}|A(x)| e^{\eta_{0}|x-y|}|B(y)| .
$$

But $\iint\left|K_{0}(x, y)\right|^{2} d x d y$ does not exceed

$$
\begin{aligned}
& \left(1 / 16 \pi^{2}\right) \int_{|x| \geqq R} \int_{|y| \geqq R}|x-y|^{-2} e^{-\left(\alpha-2 \eta_{0}\right)|x|} e^{-\left(\alpha-2 \eta_{0}\right)|y|} d x d y \\
& \quad+C_{1} \int_{|x| \leqq R} \int_{|y| \leqq R}|x-y|^{-2}|A(x)||B(y)| d x d y
\end{aligned}
$$

where $C_{1}$ is a constant. If these integrals are trivially extended to the whole space, both are seen to be convergent by Lemma 1 . Thus for Im $k>-\alpha / 2, K(x, y, k)$ is the kernel of a Hilbert-Schmidt operator, which agrees with $A R_{0}\left(k^{2}\right) B$ for $\operatorname{Im} k>0$. The analyticity statements follow from the uniform majorization by $K_{0}(x, y)$ and Lemma 2 .

If $\operatorname{Im} k>0$ and $k^{2}$ is not real, then $I+Q(k)$ has the bounded inverse $I-A\left(T-k^{2}\right)^{-1} B$ [9]. It follows by Lemma 3 that $[I+Q(k)]^{-1}$ is meromorphic for $\operatorname{Im} k>-\alpha / 2$. Finiteness of the number of poles in the upper half-plane is proved in [9].

In the present case, the $S$-matrix $S(k)$ is defined for $k>0$ and is a unitary operator on $L_{2}(\Sigma)$ where $\Sigma$ is the unit sphere in $R_{3}$ with surface measure. According to Ikebe [7, Theorem 1], $S(k)-I$ is a HilbertSchmidt operator on $L_{2}(\Sigma)$ with kernel

$$
t\left(\omega, \omega^{\prime}, k\right)=\left(-i k / 8 \pi^{2}\right) \int \phi(x, \omega, k) V(x) e^{i k \omega^{\prime} \cdot x} d x
$$

where $\omega, \omega^{\prime} \in \Sigma . \phi(x, \omega, k)$ is bounded and continuous on $R_{3}$ and satisfies

$$
\phi(x, \omega, k)=e^{-i k \omega \cdot x}-\int G_{0}(x, y, k) V(y) \phi(y, \omega, k) d y .
$$

THEOREM 2. $S(k)$ has a meromorphic continuation to $|\operatorname{Im} k|<\alpha / 2$, which has poles only at the poles of $[I+Q(k)]^{-1}$.

Proof. Let $a(x, \omega, k)=A(x) e^{-i k \omega \cdot x}$ and $b(x, \omega, k)=B(x) e^{-i k \omega \cdot x}$. Multiplying (2) by $A(x)$ and observing that $a, b$ and $A \phi$ are in $L_{2}\left(R_{3}\right)$, we find after a simple calculation that

$$
t\left(\omega, \omega^{\prime}, k\right)=\left(b(\omega, k),[I+Q(k)]^{-1} a\left(\omega^{\prime}, k\right)\right)_{L_{\mathbf{2}}\left(R_{3}\right)} .
$$

Now for each $\omega \in \Sigma, a(\omega, k)$ has an analytic continuation to Im $k>-\alpha / 2$ which is bounded in $L_{2}\left(R_{3}\right)$-norm, uniformly on 
$\Sigma \times\left\{k: \operatorname{Im} k \geqq-\eta_{0}\right\}$ for any $\eta_{0}<\alpha / 2 . b(\omega, k)$ has a similar conjugate analytic continuation to $|\operatorname{Im} k| \leqq \eta_{0}$, which is bounded uniformly on $\Sigma \times \Sigma$ by $\left\|[I+Q(k)]^{-1}\right\|$ times a constant depending on $\eta_{0}$. Analyticity in Hilbert-Schmidt norm of the kernel operator $S(k)-I$, except at poles of $[I+Q(k)]^{-1}$ now follows from Lemma 2 .

If $\operatorname{Im} k>0$ and $k^{2}$ is in the resolvent set of $T$, then [6, Theorem 1] $\left(T-k^{2}\right)^{-1}$ has a kernel $G(x, y, k)$ of Carleman type, which satisfies

$$
G(x, y, k)=G_{0}(x, y, k)-\int G_{0}(x, \xi, k) V(\xi) G(\xi, y, k) d \xi
$$

for a.e. fixed $y$. Multiplying (4) by $A(x)$, we find that the function $g(x, y, k)=A(x) G(x, y, k)$ satisfies

$$
g(\cdot, y, k)=[I+Q(k)]^{-1} g_{0}(\cdot, y, k)
$$

for a.e. fixed $y$, where $g_{0}(x, y, k)=A(x) G_{0}(x, y, k)$. Since $g_{0}(\cdot, y, k)$ has an obvious continuation to Im $k>-\alpha / 2$ as an element of $L_{2}\left(R_{3}\right),(5)$ gives a meromorphic continuation of $g(\cdot, y, k)$. Recalling that $A(x)=e^{-\alpha|x| / 2}$ yields

Theorem 3. Let $\mathcal{H C}=L_{2}\left(\mu, R_{3}\right)$ where $d \mu=e^{-\alpha|x|} d x$. Then $G(\cdot, y, k)$ has an $\mathfrak{F}$-valued meromorphic continuation to $\operatorname{Im} k>-\alpha / 2$, which has poles only at the poles of $[I+Q(k)]^{-1}$.

REMARKs. (1) In Theorems 2 and 3, we assert only that any pole of $S(k)$ or $G(\cdot, y, k)$ must also be a pole of $[I+Q(k)]^{-1}$; the converse is not established.

(2) Lemma 3 is by no means the best such result. A generalization to $Q(k)$ compact on a Hilbert space follows from [3, VII 6.13, p. 592] and [2, Appendix, Theorem 18]. Moreover, the referee has kindly informed us that the lemma has been proved for $Q(k)$ compact on a Banach space by Stanley Steinberg of Purdue and, for a separable Banach space with basis, by Peter Werner and H. Haf of Technische Hochschule, Stuttgart. These papers should appear in print soon, and are presently available as preprints.

(3) Finally, we remark that the method employed here should be generally useful in studying $S$-matrix analyticity, provided that (a) the operator $A R_{0}(z) B$ is compact and can be continued across the real axis, and (b) one has a suitable representation of the $S$-matrix in terms of the boundary values of this operator.

\section{REFERENCES}

1. L. Bers, F. John and M. Schechter, Partial differential equations, Lectures in Applied Mathematics, Vol. III, Interscience, New York, 1964. 
2. L. DeBranges and J. L. Rovnyak, "Canonical models in quantum scattering theory" in Perturbation theory and its applications to quantum mechanics, ed. C. H. Wilcox, Wiley, New York, 1966.

3. N. Dunford and J. Schwartz, Linear operators, Parts I, II, Interscience, New York, 1958, 1963.

4. C. L. Dolph, J. B. McLeod and D. Thoe, The analytic continuation of the resol vent kernel and scattering operator associated with the Schroedinger operator, J. Math. Anal. Appl. 16 (1966), 311-332.

5. J. S. Howland, On the essential spectrum of Schroedinger operators with singular potentials, Pacific J. Math. 25 (1968), 533-542.

6. T. Ikebe, Eigenfunction expansions associated with the Schroedinger operators and their applications to scattering theory, Arch. Rational Mech. Anal. 5 (1960), 1-34.

7. - On the phase-shift formula for the scattering operator, Pacific J. Math. 15 (1965), 511-523.

8. T. Kato, Fundamental properties of Hamiltonian operators of Schroedinger type, Trans. Amer. Math. Soc. 70 (1961), 195-211.

9. R. Konno and S. T. Kuroda, On the finiteness of perturbed eigenvalues, J. Fac. Sci. Univ. Tokyo Sect. I 13 (1966), 55-63.

10. J. B. McLeod, The analytic continuation to the unphysical sheet of the resolvent kernel associaied with the Schroedinger operator, Quart. J. Math. 18 (1967), 219-231.

UNIVERSITY OF VIRGINIA 